\title{
Overlapping areas of neuronal activation after motor and mental imagery training
}

\author{
Kenneth Hugdah/* \\ Department of Biological and Medical Psychology, University of Bergen and Division of Psychiatry, Haukeland University Hospital, Bergen, Norway \\ *Correspondence: hugdah@psybp.uib.no
}

\section{A commentary on}

Learning by doing and learning by thinking: an fMRI study combining motor and mental training

by Carl-Johan Olsson, Bert Jonsson and Lars Nyberg

The question whether mental imagery of perceptual and/or motor acts produces activation in the same or overlapping brain areas has fascinated neuroscientists and cognitive psychologists for several decades. For example, in a series of pioneering studies on visual imagery, Kosslyn and colleagues showed that visual imagery is associated with activity in striate and extrastriate cortex (Kosslyn and Thompson, 2003; Kosslyn et al., 2001), with the same areas being activated in visual object perception. Similarly, Ersland et al. (1996) reported in a functional magnetic resonance imaging (fMRI) study that mental imagery of a motor act, finger-tapping in a patient with an amputated right arm produced activation in contralateral motor cortex, in the same areas similar to tapping with the intact left arm fingers. In a followup study, Hugdahl et al. (2001; see also Rosen et al., 2001) revealed that imagining finger-tapping with imagined pain shifted the activation to sensory cortex areas and in sub-cortical pain pathways, such as the thalamus and insula.

Hence, it is clear that visual imagery of both object perception and motor acts has neuronal correlates that overlap with areas activated during canonical visual perception and motor execution (see also Setiz et al., 1990; Tootell et al., 1998). However, while the practice of motor skills produce activations in cerebellum, imagery learning produces activation in occipital areas such as primary and secondary visual areas (Nyberg et al., 2006). An interesting question, addressed by Olsson et al. (2008), is therefore whether combining physical and mental motor learning and practice would result in both facilitated performance and whether such a combination would produce activations in the same or different brain areas. An applied perspective of such an approach is that mental training is often used in athletics, which is combined with actual practice of the behavior in question. In a previous study by the research group (Nyberg et al., 2006) it was however suggested that combining physical and imagery training may result in interference effects, actually decreasing performance efficiency.

Olsson et al. (2008) used a novel approach of combining mental and physical motor training and evaluated the effects with fMRI, also using a novel and untrained motor act as a test for transfer effects of the training exercises. The task was a finger-tapping task in which the subjects had to practice over a 6-week period, using canonical motor training, visual imagery, or combined motor and imagery training. The transfer-of-learning task was a sequence tapping task in which the subjects had to execute a tapping sequence, after specific instructions.

Two alternative hypotheses were tested. One hypothesis was that motor and mental training would result in better performance with the largest gain observed for motor training, and that extended motor or mental training would result in increased activation in motor regions and in visual areas, respectively. On combining motor and mental training, it was further hypothesized that the training effect would be based on similar neural networks as seen after motor and mental training in isolation. The other hypothesis was that combined training would not result in better performance above the level obtained through motor training only (possibly also resulting in decreased performance due to interference effects). Such interference effects might be translated into lowered training-related fMRI activations in motor and visual brain areas.
The protocol involved three phasespre-test, training and post-test. During pre-test, the participants were instructed that each of the four fingers of the left hand represented a single digit number, where the index finger was number one and little finger was number four. The participants were then told to, as accurate and as fast as possible, sequentially tap the fingers against a laptop keyboard using F G H J according to a sequence of digits presented on a computer screen placed in front of the participants. The participants were asked to continue the tapping as long as the sequence appeared on the screen. Each sequence shown on a PC screen that the subject had to follow was shown in blocks of $30 \mathrm{~s}$. During the training phase the motor training group practiced execution of the motor sequences while the mental training group was instructed to use an imagery perspective where he should try to engage in himself as to "feel" as if the sequence was executed without actually moving the fingers. During the post- testing phase, the subjects were tested on both the old sequence and a new sequence to assess training and transfer effects. The post-testing was performed according to the same protocol as pre-test.

The results showed that all three training approaches produced better performance after training. However, mental imagery training did not produce the same increase in performance when compared to the other two training procedures. The fMRI brain activation results showed that training resulted in significant activations in ventral pre-motor cortex following motor training and in fusiform gyrus following mental training. Combined motor and mental training resulted in combined activation of motor and visual areas. The fMRI results also revealed effects of transfer of the training that showed activation in the cerebellum.

The authors conclude that combined motor and mental training recruited both motor and visual systems, and that combined motor and mental training improved 
transfer of learning possibly through setting new functional connections between cortex and the cerebellum. Although the study by Olsson et al. (2008) is limited to the training of an elementary motor act, finger tapping, the combined training effect of physical and mental training, and the transfer effect could have important consequences for training protocols for athletes in need of improved performance in, e.g., jumping or running, and for stroke patients participating in rehabilitation training programs.

\section{REFERENCES}

Ersland, L., Rosen, G., Lundervold, A., Smievoll, A. I., Tillung, T., Sundberg, H., and Hugdahl, K. (1996). Phantom limb "fingertapping" causes primary motor cortex activation: An fMRI study. Neuroreport 8, 207-210.
Hugdahl, K., Rosen, G., Ersland, L., Lundervold, A., Smievoll, A. I., Barndon, R., and Thomsen, T. (2001). Common pathways in mental imagery and pain perception: an fMRI study of a subject with an amputated arm. Scand. J. Psychol. 42, 269-275.

Kosslyn, S. M., Ganis, G., and Thompson, W. L. (2001). Neural foundations of imagery. Nat. Rev. Neurosci. 2, 635-642.

Kosslyn, S. M., and Thompson, W.L. (2003). When is early visual cortex activated during visual mental imagery. Psychol. Bull. 129, 723-746.

Nyberg, L., Eriksson, J., Larsson, A., and Marklund, P. (2006). Learning by doing versus learning by thinking: an fMRI study of motor and mental training. Neuropsychologia 44, 711-717.

Olsson, C.-J., Jonsson, B., and Nyberg, L. (2008). Learning by doing and learning by thinking: an fMRI study combining motor and mental training. Front. Hum. Neurosci. 1. doi: 10.3389/neuro.09.005.2008.

Rosen, G., Hugdahl, K., Ersland, L., Lundervold, A., Smievoll,A.I., Barndon, R., Sundberg,H., Thomsen, T., Roscher, B. E., Tjølsen, A., and Engelsen, B. (2001).
Different brain areas activated during imagery of painful and non-painful "finger movements" in a subject with an amputated arm. Neurocase 7, 255-260.

Setiz, J., Roland, P., Bohm, C., Greitz, T., and StoneElander, S. (1990). Motor learning in man: a positron emission tomography study. Neuroreport 1, 57-60.

Tootell, R. B. H., Hadjikhani, N. K., Mendola, J. D., Marrett, S., and Dale, A. M. (1998). From retinotopy to recognition: fMRI in human visual cortex. Trends Cogn. Sci. 2, 61-201.

Received: 17 February 2009; published: 01 May 2009 Citation: Front. Neurosci. (2009) 3,1: 5-6. doi: 10.3389/ neuro.01.008.2009

Copyright $\odot 2009$ Hugdahl. This is an open-access publication subject to an exclusive license agreement between the authors and the Frontiers Research Foundation, which permits unrestricted use, distribution, and reproduction in any medium, provided the original authors and source are credited.

\section{The emotional consequences of being distracted}

\section{Francisco Barceló*}

Clinical Neuropsychology Research Group, Universitat de les Illes Balears, Palma de Mallorca, Spain

*Correspondence: f.barcelo@uib.es

\section{A commentary on}

Response inhibition is linked to emotional devaluation: behavioral and electrophysiological evidence

by Monica Kiss, Jane E. Raymond, Nikki Westoby, Anna C. Nobre and Martin Eimer

Emotionally salient events have long been shown to engage attentional resources more than emotionally neutral events (Vuilleumier, 2005). In contrast, the reciprocal effect that attention also influences emotion has remained mostly unexplored in spite of everyday intuition. Imagine yourself sitting at your desk, your thoughts immersed in formulating some complex brain theory. Suddenly, the door opens and an unknown face sticks out asking: $D r$. Peters? - No, wrong office - you reply. Even if the smiling face quickly retreats in silence, it already captured your attention away, and briefly interrupted the smooth flow of your thoughts and actions. Some may find this distractor effect displeasing, particularly while handling a difficult problem, or if the interruption occurs at frequent intervals. In a research article published in Frontiers in Human Neuroscience, Kiss et al. (2008) have made an original contribution to the field by means of a novel methodology to examine the links between attentional selection and emotional valuation.

The authors tested the hypothesis that human faces would be emotionally devalued and judged as less trustworthy as a consequence of having been designated as distractors - hence requiring no overt motor response - in a previous selective attention procedure. Visual targets and distractors consisted of exemplars of human faces with distinct racial features, i.e., Caucasian versus Asian faces. Trial blocks involved two stages. Firstly, participants saw a short series of novel faces, to which they responded by pressing a button to faces of one race, and refrained from responding to faces of the other race (Figure 1). Secondly, about half-a-minute later, the same faces were judged for trustworthiness in a 4-point rating scale. Each face appeared only twice, once at response selection, and once at the affective evaluation stage, to control for familiarity effects. The response assignment of target (Go) and distractor
(Nogo) racial features was reversed halfway through the experiment.

Importantly, the neural substrates of distractor devaluation were also examined. As an index of prefrontal inhibitory control, the authors measured a negative-going brain potential peaking between 250 and $350 \mathrm{~ms}$ after the onset of Nogo distractors (often termed "Nogo N2"). The phasic negativity peaks at midfrontal scalp regions and has been considered as an electrophysiological correlate of anterior cingulate function (Nieuwenhuis et al., 2003). The intensity of Nogo N2 potentials to distractor faces was larger for faces that were rated as less trustworthy than for faces judged more positively. This indicated that the efficiency of prefrontal inhibitory control triggered by distractor faces covaried with their subsequent affective devaluation.

The phenomenon of distractor devaluation was explained from a general inhibition-based account by which the same type of inhibitory tagging responsible for top-down cognitive control could be generalized to emotional as well as "perceptual, higher cognitive, or response-related stages of processing" (Kiss et al., 2008). The 